\title{
Identification of leptospiral flagellar antigens by gel electrophoresis and immunoblotting
}

\author{
J. S. KELSON, B. ADLER, A. J. CHAPMAN and S. FAINE
}

Department of Microbiology, Monash University, Clayton 3168, Melbourne, Australia

\begin{abstract}
Summary. Flagella extracted from five serovars, representative of the pathogenic and saprophytic species of the Leptospiraceae, were morphologically similar. Analysis of Leptospira interrogans flagellar preparations by polyacrylamide gel electrophoresis revealed three common major bands in the $(30-40) \times 10^{3}$-mol. wt region, and serovarspecific bands in the lower region of the gels. Although some differences were observed, flagella extracted from L. biflexa serovar patoc and Leptonema illini revealed similar electrophoretic profiles to those seen in $L$. interrogans flagella. Immunoblot analysis showed that while flagellar components in the $(20-30) \times 10^{3}$-mol. wt region were recognised only by homologous rabbit antisera, a major protein doublet of (33$34) \times 10^{3}$ or $(35-36) \times 10^{3}$-mol. wt, depending on the species, was also demonstrated by heterologous antisera. The serovar-specific bands in the $(20-30) \times 10^{3}$-mol. wt region were composed of lipopolysaccharide (LPS). These results show that leptospiral flagella are immunogenic and contain antigens which are conserved among the different genera of the family Leptospiraceae.
\end{abstract}

\section{Introduction}

The genus Leptospira is divided into two species, $L$. interrogans, which includes pathogenic serovars and the saprophytic L. biflexa. A third species, originally classified as Leptospira illini (Faine, 1982), has now been reclassified as Leptonema illini on the basis of morphological and DNA differences (Hovind-Hougen, 1979).

Each leptospire possesses two flagella (axial filaments) inserted subterminally into the protoplasmic cylinder. The filament, sheath, hook and basal body of leptospiral flagella are similar to the corresponding structures of other bacterial flagella (Smibert, 1973). Chang and Faine (1970) showed that purified leptospiral flagella were immunogenic and on immunodiffusion formed a characteristic precipitation line. The pattern of cross-reactivity between serovars by immunodiffusion was different from that seen in the agglutination-absorption system used for classification (Chang et al., 1974).

Nauman et al. (1969) identified six protein bands when acid-dissociated leptospiral flagella were subjected to polyacrylamide gel electrophoresis (PAGE). In contrast, other bacterial flagella usually possessed a single band. However, the antigenicity of these protein components was not studied, nor was their flagellar origin proved. There have been

Received 19 May 1987; accepted 7 Aug. 1987. no further studies on either the characteristics or antigenicity of leptospiral flagellar components.

In this present study, the composition of flagella prepared from serovars representative of each of the three species in the family Leptospiraceae was investigated by sodium dodecyl sulphate-polyacrylamide gel electrophoresis (SDS-PAGE), followed by immunoblotting to determine their immunogenicity and cross-reactivity.

\section{Materials and methods}

\section{Organisms, antigens, antibodies and serological methods}

The growth and culture of leptospires and the preparation of rabbit antisera against whole, live leptospires have been described previously (Chang and Faine, 1970; Adler and Faine, 1978).

Antiserum was prepared against SDS-PAGE separated flagella components by the method of Boulard and Lecroisey (1982). Coomassie blue stained bands were excised from the gel, macerated and emulsified with incomplete Freund's adjuvant $(1: 1 \cdot 2 \mathrm{v}: \mathrm{v})$. Female New Zealand White rabbits were given intradermal injections of this material at approximately five sites and bled 4 weeks later. The production and characterisation of monoclonal antibodies reacting with homologous LPS epitopes of serovars hardjo and pomona were performed as described previously (Adler and Faine, 1983; Farrelly et al., 1987).

The microscopic agglutination test (MAT) was de- 
scribed by Adler and Faine (1983). Flagella, purified by differential centrifugation followed by caesium chloride gradient centrifugation, were negatively stained (Chang and Faine, 1970; Adler and Faine, 1979) and examined with a Philips EM 300 electronmicroscope at an accelerating voltage of $80 \mathrm{kV}$. Flagella were stained with gold labelled anti-rabbit immunoglobulins (Janssen auroprobe EMGAR, $5 \mathrm{~nm}$ diameter) by the procedure described by Barbour et al. (1984) with the following modifications. Grids were incubated with antibody at $37^{\circ} \mathrm{C}$ in a humid atmosphere, Tween 20 (Sigma) $0.05 \%$ was added to the washing buffer and grids were washed with distilled water before negative staining.

\section{Electrophoresis and immunoblotting}

SDS-PAGE analysis of the purified flagellar preparations was performed with the discontinuous buffer system of Laemmli (1970) with resolving and stacking gels containing polyacrylamide $10 \%$ and $4 \%$ respectively. Flagellar protein $5 \mu \mathrm{g} /$ lane was loaded on to the gels and electrophoresed at $30 \mathrm{~mA} / \mathrm{gel}$, together with low mol. wt markers (Pharmacia Fine Chemicals, Sweden). After electrophoresis, the gels were either silver-stained (Morrissey, 1981) or the separated flagellar proteins were transferred to nitrocellulose $(0.45 \mu \mathrm{m}$ pore size; Schleicher and Schuell Co.) with a Biorad Transblot Cell (Biorad, USA) run at $60 \mathrm{~V}$ for $3 \mathrm{~h}$. The transblot buffer described by Towbin et al. (1979) was diluted 1 in 2 to facilitate transfer of high mol. wt proteins. Transblotted mol. wt markers were stained with india ink (Hancock and Tsang, 1983) to check efficiency of transfer. After transfer, antigenic components were detected by immunostaining (Chapman et al., 1987) with rabbit antisera or mouse monoclonal antibodies, followed by either peroxidase conjugated goat anti-rabbit immunoglobulins (Biorad) or sheep anti-mouse immunoglobulins (Silenus, Australia) respectively. Bands were visualised with 4chloro-1-naphthol (Hawkes et al., 1982).

\section{Results}

\section{Electronmicroscopy}

Electronmicroscopic examination of purified flagellar preparations revealed only filamentous structures (fig. 1). The filaments had a striated appearance whereas thinner, apparently unsheathed, filaments were seen occasionally. Flagellar preparations extracted from each of the five serovars tested were morphologically indistinguishable.

\section{Electrophoresis and immunostaining}

Flagellar preparations were electrophoretically separated on SDS-PAGE $10 \%$ gels and stained for protein. Except for minor variations in the mol. wts of the bands, similar profiles were obtained for flagella preparations from each serovar (fig. 2).

Flagellar preparations extracted from the $L$. interrogans serovars revealed an identical banding profile in the mol. wt region $(30-40) \times 10^{3}$, each showing major bands of approximate mol. wts $37\left(10^{3}\right)$ and $31\left(10^{3}\right)$, and a $(33-34) \times 10^{3}$-mol. wt doublet.

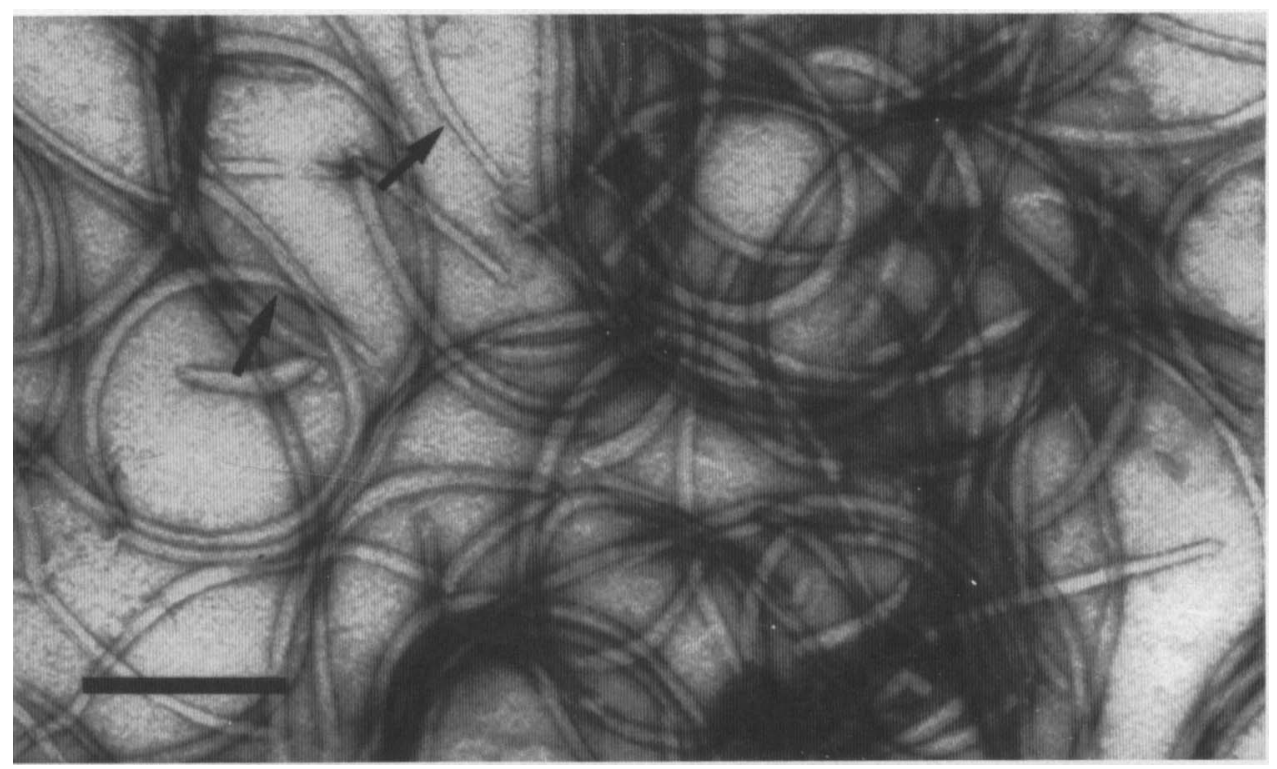

Fig. 1. Electronmicrograph of flagella extracted from $L$. interrogans serovar pomona negatively stained with uranyl acetate $0 \cdot 05 \%$. Arrows indicate partially sheathed flagella. Bar $=0 \cdot 2 \mu \mathrm{m}$. 


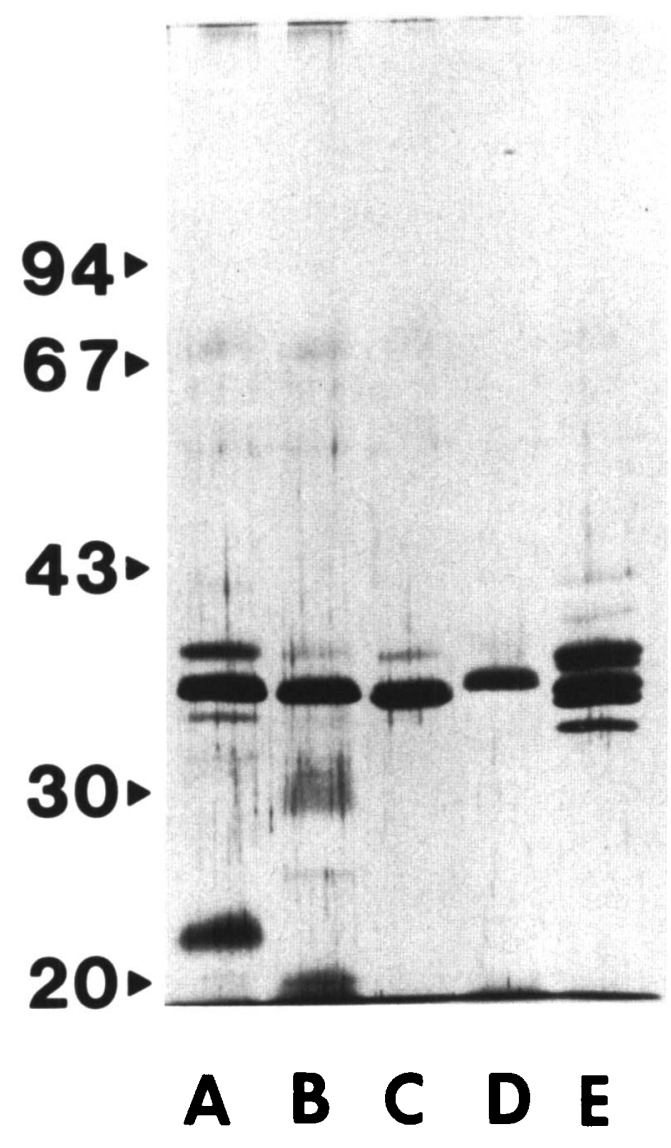

Fig. 2. Silver-stained SDS-PAGE gel of leptospiral flagellar preparations. The positions of mol. wt markers are indicated $\left(10^{3}\right)$. Lane A-L. interrogans serovar pomona; B-L. interrogans serovar hardjo; $\mathrm{C}-L$. interrogans serovar copenhageni; $\mathrm{D}-L$. biflexa serovar patoc; $\mathrm{E}-$ L. illini.

In the $(20-30) \times 10^{3}$-mol. wt region of the gel, profiles unique to each serovar were observed. Flagellar preparations from serovars pomona and hardjo had two and three additional bands respectively (fig. 2), whereas flagella from serovar copenhageni revealed no bands below mol. wt $30 \times 10^{3}$. Flagellar preparations from $L$. biflexa serovar patoc had a profile in the $(30-40) \times 10^{3}-\mathrm{mol}$. wt region similar to those seen in L. interrogans although mol. wts were slightly higher (fig. 2). In this case, the mol. wts $\left(10^{3}\right)$ of the major bands were 38,34 and the doublet 35-36. In contrast, flagella extracted from $L$. illini revealed two doublets of approximate mol. wts $\left(10^{3}\right) \quad 36-37$ and $33-34$ respectively. Additional bands of mol. wts $\left(10^{3}\right) 31,39$ and 41 were also observed (fig. 2).

After transfer to nitrocellulose, the antigenicity of the flagellar proteins was examined by probing the separated proteins with rabbit antisera. These
Table. Serological cross-reactivity of different leptospiral serovars measured by MAT with rabbit antisera

\begin{tabular}{l|rrrrr}
\hline \multirow{2}{*}{$\begin{array}{l}\text { Serovar } \\
\text { of test } \\
\text { strain }\end{array}$} & \multicolumn{5}{c}{ MAT titre with antiserum raised against serovar } \\
\cline { 2 - 6 } & pomona & hardjo & copenhageni & patoc & illini \\
\hline $\begin{array}{l}\text { pomona } \\
\text { hardjo }\end{array}$ & 512 & 8 & 0 & 2 & 0 \\
copenhageni & 0 & 1024 & 2 & 0 & 0 \\
patoc & 4 & 0 & 512 & 0 & 0 \\
illini & 0 & 0 & 0 & 256 & 8 \\
& 0 & 0 & 0 & 0 & 512 \\
\hline
\end{tabular}

sera had high homologous agglutinating titres but only very low titres against heterologous serovars (table). All antisera produced against $L$. interrogans serovars recognised the three major flagellar components extracted from other serovars of $L$. interrogans, as exemplified by the pomona antiserum (fig. 3 ), although the band of mol. wt $31 \times 10^{3}$ was only faintly detectable. The sera also reacted with bands of mol. wts $\left(10^{3}\right) \quad 38$ and $35-36$ in flagellar preparations of $L$. biflexa serovar patoc and with the $(33-34) \times 10^{3}-\mathrm{mol}$. wt doublet band of L. illini flagella. The cross-reactivity pattern was identical regardless of the $L$. interrogans serovar used to produce the antisera. Rabbit antisera to serovar patoc reacted strongly with the $(33-34) \times 10^{3}-\mathrm{mol}$. wt doublet of both $L$. interrogans and $L$. illini flagella. There was also faint reactivity with the $37 \times 10^{3}$ mol. wt band of $L$. interrogans and the (36-37) $\times$ $10^{3}$-mol wt doublet of L. illini (fig. 3). L. illini antiserum reacted with the doublet from all other serovars and with additional homologous components.

As well as recognising heterologous bands, all sera reacted with low-mol. wt serovar-specific bands, when they were present (fig. 3), which were identical to those seen in silver stained gels (fig. 2).

\section{Identity of low-mol. wt bands}

In preparations from serovars hardjo and pomona, bands in the $(20-30) \times 10^{3}-\mathrm{mol}$. wt region were immunostained with monoclonal antibodies specific for homologous LPS, producing identical profiles to those seen when purified LPS was similarly electrophoresed and stained (data not shown).

Investigation of the cross-reactive flagellar components

Rabbit antiserum prepared against the (33-34) $\times$ $10^{3}$-mol. wt doublet of flagellar preparations from 

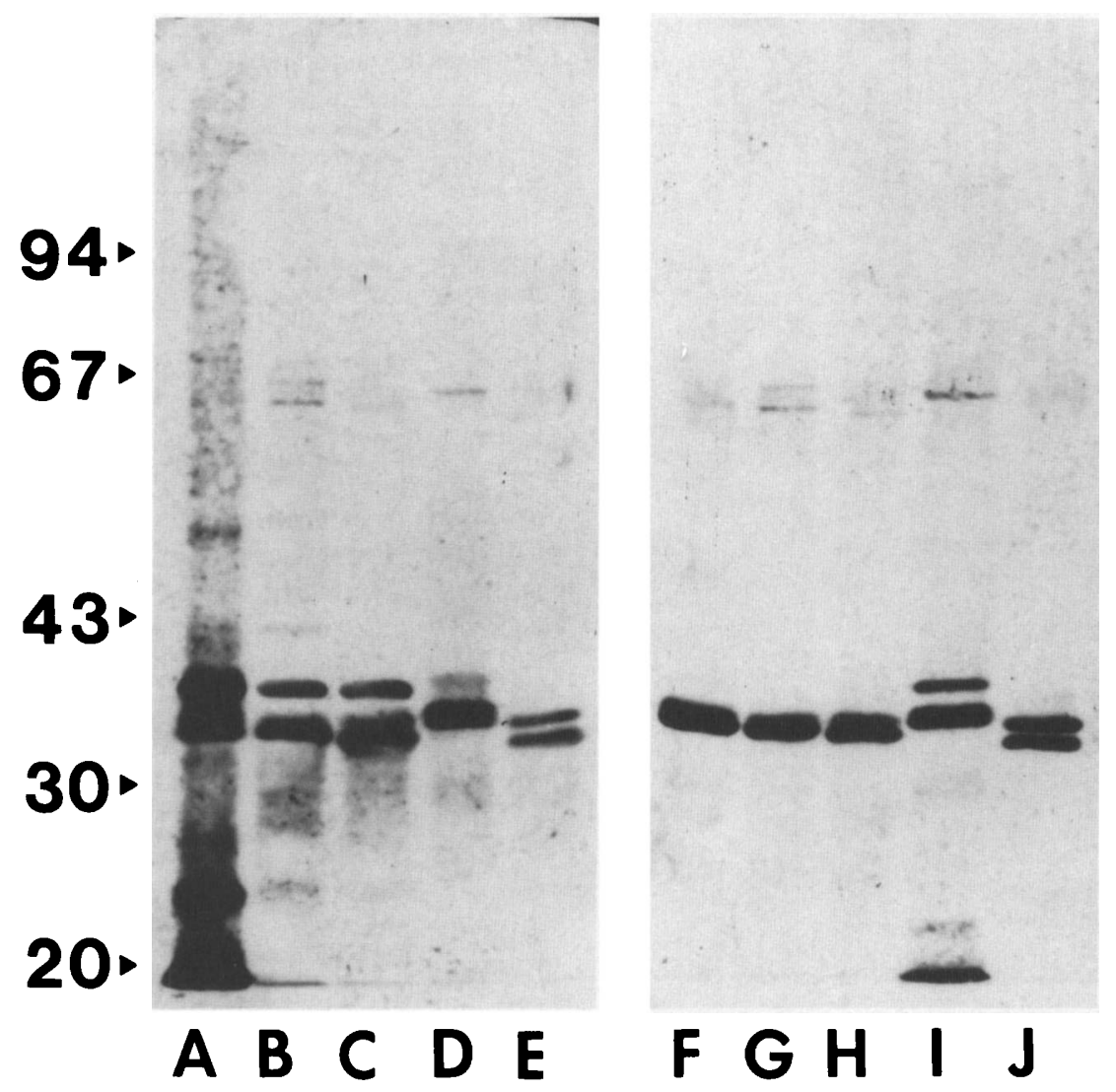

Fig. 3. Immunoblot of leptospiral flagellar preparations stained with rabbit antisera against L. interrogans serovar pomona (lanes A-E) or L. biflexa serovar patoc (lanes F-J). The positions of mol. wt markers are indicated (10). Lanes A and F-L. interrogans serovar pomona; $\mathrm{B}$ and $\mathrm{G}-L$. interrogans serovar hardjo; $\mathrm{C}$ and $\mathrm{H}-L$. interrogans serovar copenhageni $; \mathrm{D}$ and $\mathrm{I}-L$. biflexa serovar patoc; $\mathrm{E}$ and $\mathbf{J}-$ L. illini.

L. interrogans serovar pomona (anti-doublet serum) failed to agglutinate whole leptospires. Analysis by immunoblotting showed that the same antiserum reacted strongly with both the (33-34)- and 37-mol. wt $\left(10^{3}\right)$ bands of flagellar preparations of the three $L$. interrogans serovars, as well as the (35-36)- and the (33-34)-mol. wt $\left(10^{3}\right)$ doublets of $L$. biflexa serovar patoc and $L$. illini respectively (fig. 4$)$. The anti-doublet serum failed to react with transblotted, purified LPS or other cellular components of $L$. interrogans serovar pomona.

To determine whether the antigens recognised by the anti-doublet serum were indeed of flagellar origin, this antiserum was allowed to react with serovar pomona flagella and immunogold stained. The gold particles preferentially labelled the entire length of the flagella (fig. 5). In contrast, flagella similarly allowed to react with normal rabbit serum (NRS) failed to bind the gold particles (fig. 5).

\section{Discussion}

Electronmicroscopic examination of all the flagellar preparations revealed the presence of thick and thin filamentous structures. The thin filaments were probably unsheathed thick filaments, some of which had their sheaths partially removed. Similar structures have been described for flagellar preparations extracted from other leptospires (Nauman et al., 1969; Smibert, 1973) and treponemes (Bharier and Rittenberg, 1971; Sand Petersen et al., 1981). The striated appearance of the filaments has also been noted in other leptospires and treponemes (Nauman et al., 1969; Bharier et al., 1971; Jackson and Black, 1971).

Analysis of the flagellar preparations by SDSPAGE revealed similar banding profiles in the (30$40) \times 10^{3}$-mol. wt range, comprising a doublet and two satellite bands. Banding profiles identical to those reported here in the $(30-40) \times 10^{3}-\mathrm{mol}$. wt 


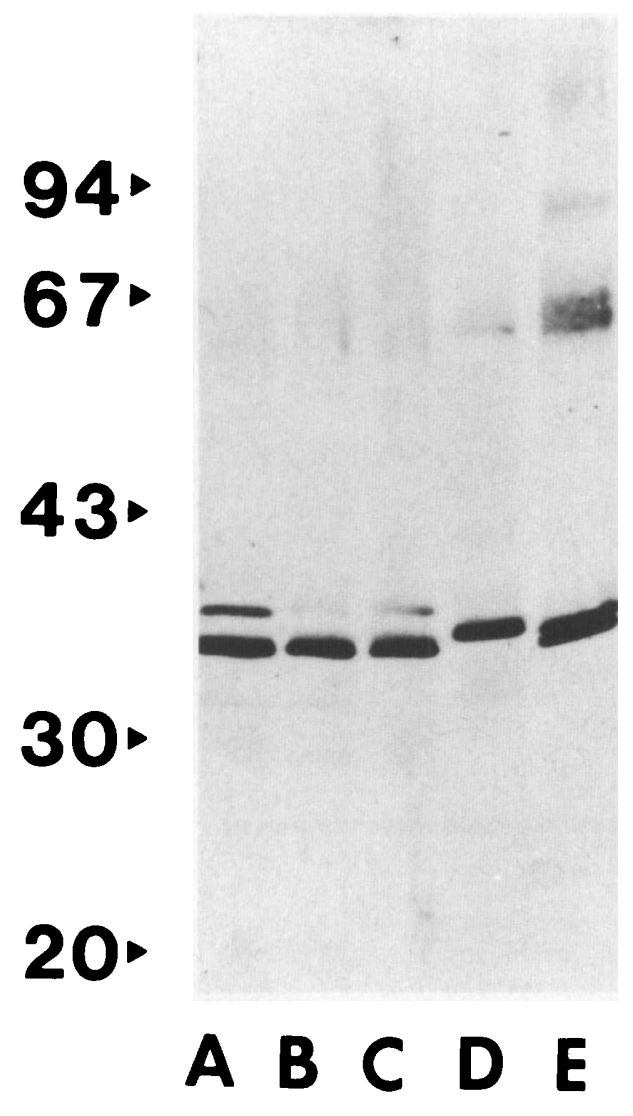

Fig. 4. Immunoblot of leptospiral flagellar preparations stained with anti-doublet serum. The positions of mol. wt markers are indicated $\left(10^{3}\right)$. Lane A-L. interrogans serovar pomona; $\mathrm{B}-L$. interrogans serovar hardjo; $\mathrm{C}-L$. interrogans serovar copenhageni; D-L. biflexa serovar patoc; E-L. illini.

range have been described for flagellar preparations extracted from treponemes (Bharier and Allis, 1974; Sand Petersen et al., 1981; Penn et al., 1985) although the mol. wt of the components differed slightly. The major protein components of Borrelia spp. flagella also appear as a doublet of either 39 or $41 \mathrm{~mol}$. wt $\left(10^{3}\right)$ depending on the species (Barbour et al., 1986).

However, unique, low-mol. wt bands - $(20-30) \times$ $10^{3}$-mol. wt range - were also observed in flagella extracted from $L$. interrogans serovars pomona and hardjo and L. biflexa serovar patoc. These bands were shown to be LPS antigens by their reactivity with LPS specific monoclonal antibodies. The association of LPS components with the flagellar structure is unclear because flagellar preparations lacking low-mol. wt components (as seen with serovar copenhageni) appeared morphologically identical to those with them. Contamination of flagellar preparations with LPS during extraction may be a possible explanation as this is commonly encountered when attempting to purify antigens from gram-negative bacteria (Gregory, 1986).

The anti-doublet serum reacted with both the (33-34) $\times 10^{3}-\mathrm{mol}$. wt doublet of serovar pomona, against which it was raised, and the band of mol. wt $37 \times 10^{3}$, suggesting either shared antigenic determinants or contamination with the $37 \times 10^{3}$ mol. wt band during excision from PAGE gels. Immuno-electronmicroscopy of pomona flagella showed that this serum reacted specifically with native flagella, demonstrating that the $(33-34) \times$ $10^{3}$-mol. wt doublet or the $37 \times 10^{3}$-mol- wt band, or both, of serovar pomona are flagellar components. In similar experiments, polyclonal rabbit antisera against denatured treponemal flagellar components failed to react with native flagella (Limberger and Charon, 1986). This may reflect differences in the extraction of the denatured proteins or the immunisation procedure from those used here. Immunoelectronmicroscopy with monoclonal antibodies has previously identified the major doublet of both treponemal and borrelial flagellar preparations to be of flagellar origin (Barbour et al., 1986; Limberger and Charon, 1986).

The extent of cross-reactivity of the major flagellar proteins, especially that of the doublet, suggests that flagella from both genera of Leptospiraceae share several antigenic determinants indicating that flagella may be a conserved structure. The cross-reactivity of leptospiral or leptonemal flagella with those from other spirochaetes was not examined. The possibility that leptospiral flagella may also cross-react serologically with other, less closely related, spirochaetes cannot therefore be excluded. However, the major flagellar protein of Borrelia spp., which was also found to cross-react within its genus, did not react with other spirochaetes (Barbour et al., 1986).

In a previous study of leptospiral flagella (Chang et al., 1974), the overall cross-reactivities between serovars and species which we have reported were not observed. In view of the relatively insensitive immunodiffusion technique used by Chang et al. (1974) compared with immunoblotting, it is possible that some cross-reactions were not detected. For example, in their study, neither serovar patoc flagella nor serovar patoc antisera reacted with serovars pomona, hardjo or copenhageni. Nor did they observe any cross-reactions between these serovars of $L$. interrogans, while we found strong reciprocal cross-reactions.

A second possibility is that because we used SDSdissociated flagella, we detected antigens or epitopes not accessible on native flagella. Thus, the 

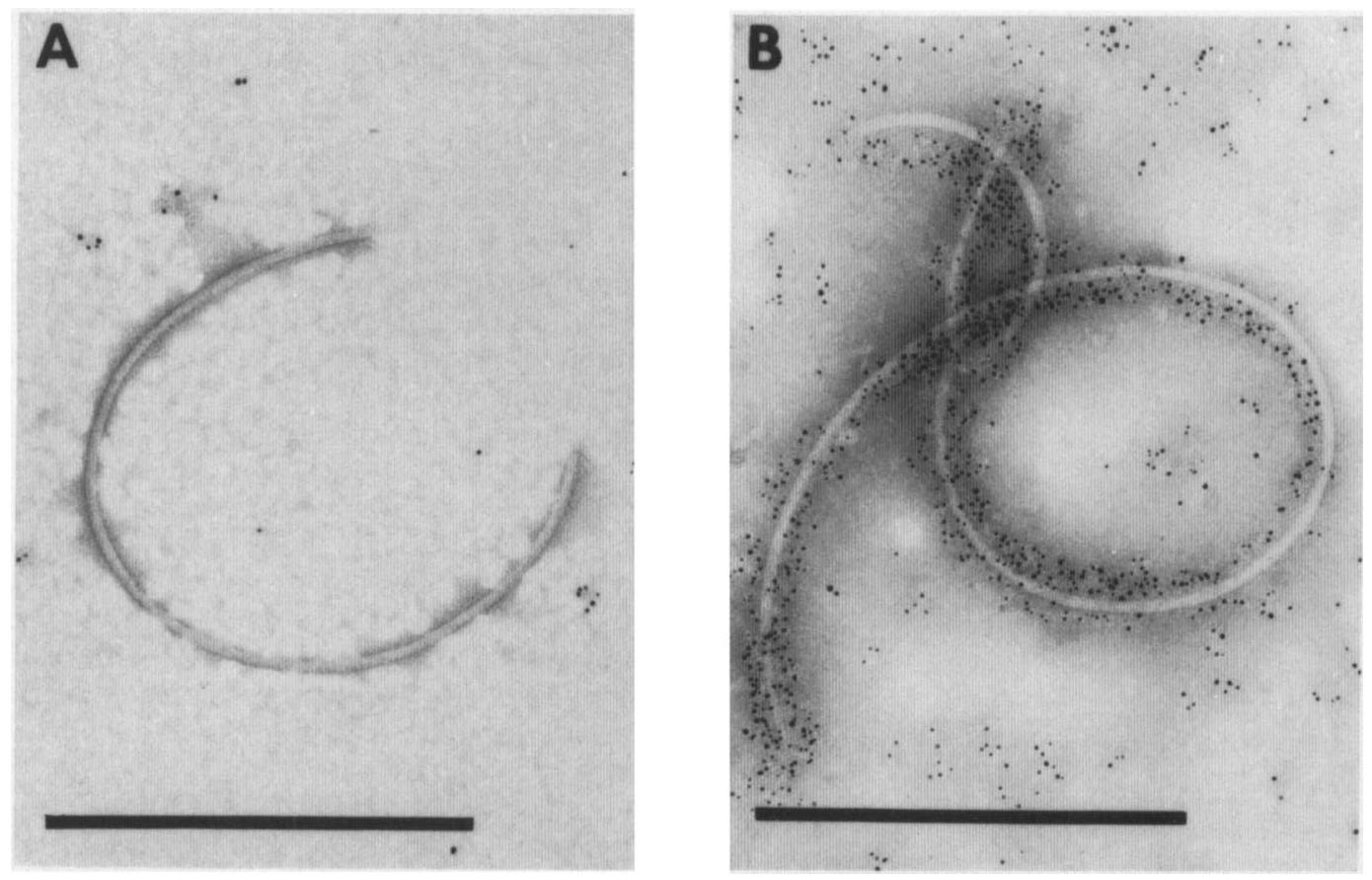

Fig. 5. Electronmicrograph of flagella extracted from L. interrogans serovar pomona stained with (A) normal rabbit serum, or (B) anti-doublet serum, followed by colloidal gold-conjugated anti-rabbit immunoglobulins. Negative staining was performed with ammonium molybdate $2 \%$. Bar $=0.5 \mu \mathrm{m}$.

doublet may represent internal flagellar antigens which are conserved throughout the family Leptospiraceae. Similar internal antigens have been described for the flagella of Vibrio spp. (Shinoda $e t$ al., 1980) and treponemes (Limberger and Charon, 1986). This second possibility is unlikely because we have shown by immuno-electronmicroscopy that the major flagellar components seen in denaturing gels are recognised on native flagella.

The lack of cross-reactivity between serovars by MAT and the high level of cross-reactivity shown by flagellar antigens in immunoblotting indicate that it is unlikely that flagella are involved in the agglutination of leptospires by immune rabbit serum. Previous reports have shown that antiflagella sera agglutinate whole leptospires (Chang and Faine, 1973). This may well have been due to contamination of flagellar preparations with LPS

\section{REFERENCES}

Adler B, Faine S 1978 The antibodies involved in the human immune response to leptospiral infection. Journal of Medical Microbiology 11 : 387-400.

Adler B, Faine S 1979 Antibodies against leptospiral axial filament in human anti-Leptospira sera. FEMS Microbiology Letters 5:57-60.

Adler B, Faine S 1983 A Pomona serogroup-specific, agglutin- which is immunogenic and can elicit the production of agglutinating antibodies (Adler and Faine, 1983; Farrelly et al., 1987). Leptospiral flagella are strongly immunogenic, being recognised by antibodies induced by natural and experimental leptospirosis in humans and rabbits (Chang and Faine, 1973; Adler and Faine, 1979) and carry antigenic determinants common to all three species. Since flagella of other gram-negative organisms are involved in antibody-mediated immunity (Holder et al., 1982; Tamura et al., 1984), further study of the role of anti-flagella antibodies in immunity to leptospirosis is warranted.

This work was supported by a grant from the National Health and Medical Research Council, Canberra, Australia. The expert assistance of $\mathbf{K}$. Hoe and $\mathbf{M}$. Goodman with the immunoelectronmicroscopy is gratefully acknowledged.

ating antigen in Leptospira, identified by monoclonal antibodies. Pathology 15:247-250.

Barbour A G, Tessier S L, Hayes S F 1984 Variation in a major surface protein of Lyme disease spirochetes. Infection and Immunity 45:94-100.

Barbour A G, Hayes S F, Heiland R A, Schrumpf M E, Tessier S L 1986 A Borrelia-specific monoclonal antibody binds to a flagella epitope. Infection and Immunity 52 : 549-554.

Bharier M A, Rittenberg S C 1971 Chemistry of axial filaments 
of Treponema zuelzerae. Journal of Bacteriology 105:422429.

Bharier M A, Eiserling F A, Rittenberg S C 1971 Electron microscopic observations on the structure of Treponema zuelzerae and its axial filaments. Journal of Bacteriology 105: 413-421.

Bharier M, Allis D 1974 Purification and characterization of axial filaments from Treponema phagedenis biotype reiterii (the Reiter treponeme). Journal of Bacteriology 120:14341442.

Boulard C, Lecroisey A 1982 Specific antisera produced by direct immunization with slices of polyacrylamide gel containing small amounts of protein. Journal of Immunological Methods 50:221-226.

Chang A, Faine S 1970 Electron microscopic evidence for reactions of axial filaments of Leptospira with IgM and IgG antibodies. Bulletin of the World Health Organization 43:571-577.

Chang A, Faine S 1973 Effect of anti-cell and anti-axial filament sera on Leptospira. The Australian Journal of Experimental Biology and Medical Science 51 :847-856.

Chang A, Faine S, Williams W T 1974 Cross-reactivity of the axial filament antigen as a criterion for classification of Leptospira. The Australian Journal of Experimental Biology and Medical Science 52:549-568.

Chapman A, Adler B, Faine S 1987 Genus-specific antigens in Leptospira revealed by immunoblotting. Zentralblatt fur Bakteriologie Mikrobiologie und Hygiene, Series A 264:279293.

Faine S (ed) 1982 Guidelines for the control of leptospirosis. WHO Offset Publication No. 67, World Health Organization, Geneva.

Farrelly H E, Adler B, Faine S 1987 Opsonic monoclonal antibodies against lipopolysaccharide antigens of Leptospira interrogans serovar hardjo. Journal of Medical Microbiology 23:1-7.

Gregory R L 1986 Microbial ribosomal vaccines Reviews of Infectious Diseases 8:208-217.

Hancock K, Tsang V C W 1983 India ink staining of proteins on nitrocellulose paper. Analytical Biochemistry 133:157162.

Hawkes R, Niday E, Gordon J 1982 A dot-immunobinding assay for monoclonal and other antibodies. Analytical Biochemistry 119: 142-147.

Holder I A, Wheeler R, Montie T C 1982 Flagella preparations from Pseudomonas aeruginosa: animal protection studies. Infection and Immunity 35:276-280.

Hovind-Hougen K 1979 Leptospiraceae, A new family to include Leptospira Noguchi 1917 and Leptonema gen. nov. International Journal of Systematic Bacteriology 29:245-251.

Jackson S, Black S H 1971 Ultrastructure of Treponema pallidum Nichols following lysis by physical and chemical methods. II Axial filaments. Archiv für Mikrobiologie 76:325-340.

Laemmli U K 1970 Cleavage of structural proteins during the assembly of the head of bacteriophage T4. Nature 227:680685.

Limberger R J, Charon N W 1986 Treponema phagedenis has at least two proteins residing together on its periplasmic flagella. Journal of Bacteriology 166: 105-112.

Morrissey J H 1981 Silver stain for proteins in polyacrylamide gels : a modified procedure with enhanced uniform sensitivity. Analytical Biochemistry 117:307-310.

Nauman R K, Holt S C, Cox C D 1969 Purification, ultrastructure and composition of axial filaments from Leptospira. Journal of Bacteriology 98:264-280.

Penn C W, Bailey M J, Cockayne A 1985 The axial filament antigen of Treponema pallidum. Immunology 54:635-641.

Sand Petersen C, Standberg Pedersen N, Axelsen N H 1981 A simple method for the isolation of flagella from Treponema Reiter. Acta Pathologica Microbiologica et Immunologica Scandinavica C89: 379-385.

Shinoda S, Senoh T, Asano K, Nakahara N, Ono B 1980 Differences between surface antigenic determinants of polar monotrichous flagella of Vibrio parahaemolyticus and of related species. Microbiology and Immunology 24:409418.

Smibert R M 1973 Spirochaetales, a review. C.R.C. Critical Reviews in Microbiology 2:491-552.

Tamura Y, Minamoto N, Tanaka S 1984 Demonstration of protective antigen carried by flagella of Clostridium chauvoei. Microbiology and Immunology 28: 1325-1332.

Towbin H, Staehelin T, Gordon J 1979 Electrophoretic transfer of proteins from polyacrylamide gels to nitrocellulose sheets : procedure and some applications. Proceedings of the National Academy of Science of the USA 76:4350-4354. 